\title{
金属液体射流变形的光滑粒子流体动力学模拟
}

\author{
马 利 王双连 郭乙木 \\ (浙江大学力学系, 杭州 310027. E-mail: mali@ustc.edu)
}

\begin{abstract}
摘要 采用光滑粒子流体动力学方法 $(\mathrm{SPH})$ 模拟液体射流与刚性表面的碰撞, 建立了半径为 $0.24 \mathrm{~cm}$, 高为 $0.75 \mathrm{~cm}$ 的圆柱形铝液滴以 $100 \mathrm{~m} / \mathrm{s}$ 的速度与刚性表面发生碰撞的 $\mathrm{SPH}$ 计算模型. 在计算中借助 Voronoi 多边形分配粒子的初始质量, 并考虑了大变形过程中随着粒子相互间距离的变化而采用可变光 滑半径. 根据计算结果分析了碰撞过程中圆柱形液滴沿轴向的压力波形演化特征, 并给出了不同时刻 金属液体射流的变形情况. 通过粒子速度随时间的变化曲线, 观察到液体在碰撞后首先进入一个稳定 流动的过程, 此稳定流动的过程相对较短, 随后流动开始发生混乱.
\end{abstract}

关键词 SPH 射流变形 碰撞

高速液体与固体表面的碰撞广泛地存在于自然 界和工业生产活动中，例如水射流切割技术就是利 用高压水从精细小孔射出，从而产生强大的切割力 来切割金属或复合材料. 这是一种典型的液体高速 冲击固体的现象. 此外, 在金属的熔化、浇注等过程 中, 也存在液体金属与固体模具或容器之间的相互 作用.

以上所提到的这些液体与固体的碰撞实际上都 是一个很复杂的过程, 除了碰撞本身所包含的界面 接触问题外, 还同时涉及液体与固体的力学性质, 并 包含一系列大变形、断裂等计算上较难处理的问题.

虽然在流体与固体领域已各自发展了成熟的数 值计算方法, 如有限差分法和有限元法等, 但应用有 限元法处理上述的液体高速冲击固体问题时, 由于涉 及到极度大变形如液体的飞溅和固体的断裂等, 在这 过程中可能会引起网格的严重扭曲或失效, 从而影响 计算的精度. 即使基于任意拉格朗日-欧拉(arbitrary Lagrange-Eulerian formulation)描述, 要对一个复杂的 三维问题不断进行网格的重构以及接触界面的识别 和处理, 在计算上仍然是很困难的.

光滑粒子流体动力学方法 (smoothed particle hydrodynamics, SPH) 是近 20 多年来逐步发展起来的一 种无网格方法，该方法的基本思想是将连续的流体 (或固体)用相互作用的质点组来描述, 各个物质点上 承载各种物理量, 包括质量和速度等, 通过求解质点 组的动力学方程和跟踪每个质点的运动轨道, 求得 整个系统的力学行为. 这类似于物理学中的粒子云 (particle-in-cell)模拟. 从原理上说, 只要质点的数目
足够多, 就能精确地描述力学过程.

虽然在 SPH 方法中, 解的精度也依赖于质点的 排列, 但它对点阵排列的要求远远低于网格的要求. 由于质点之间不存在网格关系, 因此它可避免极度 大变形时网格扭曲而造成的精度破坏等问题, 并且 也能较为方便的处理不同介质的交界面. SPH 的优点 还在于它是一种拉格朗日方法, 能避免欧拉描述中 欧拉网格与材料的界面问题, 因此特别适合于求解 高速碰撞等动态大变形等问题.

最初的 SPH方法是 1977 年首次由 Lucy ${ }^{[1]}$ 和 Gingold等人 ${ }^{[2]}$ 分别提出的. Johnson等人 ${ }^{[3]}$ 提出了归 一化的光滑函数算法, 这一算法能够提高 SPH的计算 精度, 并能通过分片试验. Johnson等人 ${ }^{[4,5]}$ 还将 $\mathrm{SPH}$ 法应用于圆杆的撞击以及弹体侵彻的数值计算, 得 到了很好的结果.

通常水射流切割过程中往往添加磨料粒子, 水 射流作为载体使磨料粒子加速, 从而产生切割力. 对 于这样一种非均匀的混合悬浮液, 难以作为一般连 续介质确定其材料属性及参数. 因此, 我们选用液态 铝作为射流材料, 考察铝液滴冲击刚性平面的过程, 并且较之水射流而言, 铝液射流在经历碰撞及后续 的流动中各阶段层次明显, 这对应用 SPH方法模拟 各种液体冲击固体过程具有典型意义. 在计算中使 用 Voronoi 多边形分配初始粒子质量, 并考虑了大变 形过程中随着粒子相互间距的变化而采用可变光滑 半径. 本文分析了铝液柱与刚性平面发生碰撞并进 入稳定流动的过程, 并给出了金属液体射流在各时 刻的变形图像. 


\section{$1 \mathrm{SPH}$ 基本理论}

SPH 方法的核心思想是用一个作用在近程范围 内的核函数描述空间的粒子及其邻粒子的相互关系. 设 $F(x)$ 为一场函数, 则

$$
F^{h}\left(x_{I}\right)=\int_{\Omega} w\left(x_{I}-x_{J}, h\right) F\left(x_{J}\right) \mathrm{d} \Omega_{J},
$$

其中 $w\left(x_{I}-x_{J}, h\right)$ 是核函数, $h$ 是核函数的影响半径, 关于核函数应满足的条件可参见文献[2].

将上式转化为离散化求和形式:

$$
F\left(x_{I}\right)=\sum_{J=1}^{N} w_{I J} \frac{m_{J}}{\rho_{J}} F\left(x_{J}\right)
$$

并可得

$$
\nabla F\left(x_{I}\right)=\sum_{J=1}^{N} \frac{m_{J}}{\rho_{J}} \nabla w_{I J} F\left(x_{J}\right),
$$

对密度场应用(2)式, 则

$$
\rho\left(x_{I}\right)=\sum_{J=1}^{N} w_{J} m_{J} .
$$

在流体动力学中, 可用如下一组方程组来描述流体 的运动和状态:

$$
\begin{cases}\nabla \cdot \sigma=\rho \dot{v} & \text { (运动方程) } \\ \dot{\rho}+\rho \nabla \cdot v=0 & \text { (连续方程), } \\ \rho \dot{E}=\sigma \cdot \dot{\varepsilon} & \text { (能量方程) }\end{cases}
$$

对于(5)式中的运动方程, 应用 SPH 方法并加以离散 化得

$$
\dot{v}_{I}=\frac{1}{\rho_{I}} \sum_{J=1}^{N} \frac{m_{J}}{\rho_{J}} \sigma_{J} \nabla w_{I J},
$$

(5)式通过一定形式的变形得到其对称形式:

$$
\dot{v}_{I}=\sum_{J=1}^{N} m_{J}\left(\frac{\sigma_{I}}{\rho_{I}^{2}}+\frac{\sigma_{J}}{\rho_{J}^{2}}\right) \nabla w_{I J} .
$$

根据(5)式, 可由 $t^{n}$ 时刻的应力求得 $t^{n}$ 时刻的加速 度, 进而根据显式中心差分格式:

$$
\begin{aligned}
& v_{I}^{n+1 / 2}=v_{I}^{n-1 / 2}+\frac{1}{2}\left(\Delta t^{n+1 / 2}+\Delta t^{n-1 / 2}\right) \dot{v}_{I}^{n}, \\
& x_{I}^{n+1}=x_{I}^{n}+\Delta t^{n+1 / 2} v_{I}^{n+1 / 2},
\end{aligned}
$$

求出 $t^{n+1}$ 时刻的位移与速度, 从而得到 $t^{n+1 / 2}$ 时刻的应 变率:

$$
\dot{\varepsilon}_{I}^{n+1 / 2}=\frac{1}{2}\left[\nabla v+(\nabla v)^{\mathrm{T}}\right]_{I}^{n+1 / 2} .
$$

对于(5)式中的连续方程, 类似以上推导, 有

$$
\rho_{I}^{n+1}=\rho_{I}^{n}+\Delta t^{n+1 / 2}\left\{\sum_{J=1}^{N} m_{J}\left(v_{I}-v_{J}\right) \cdot \nabla w_{I J}\right\}_{\text {encichin }}^{n+1 / 2}
$$

再结合流体的本构方程和能量方程, 可得 时刻的 应力 和内能

$\mathrm{SPH}$ 常用的核函数是 $\mathrm{B}$ 样条函数, 但是 $\mathrm{B}$ 样条函 数由于其一阶和二阶导数在支撑域内都不是单调递 减的, 通常会引起张应力不稳定现象 ${ }^{[4,6]}$. 根据文献 [4]的讨论, 这里选用性能较优的Quadratic光滑函数:

$$
w_{I J}=\frac{1}{\pi h_{I J}^{3}}\left(\frac{3}{8} r_{I J}^{2}-\frac{3}{2} r_{I J}+\frac{3}{2}\right), \quad 0 \leqslant r \leqslant 2,
$$

其一阶导数为

$$
w_{I J}^{\prime}=\frac{1}{\pi h_{I J}^{3}}\left(\frac{3}{4} r_{I J}-\frac{3}{2}\right), \quad 0 \leqslant r \leqslant 2,
$$

其中 $h$ 是核函数作用范围的尺寸度量, 也称为光滑长 度, $r$ 是粒子间距与光滑长度 $h$ 的比值.

\section{2 可变光滑长度}

对于复杂流体大变形过程, 粒子的相互距离在 每一时间步都发生很大的变化, 因此计算过程中必 须考虑随粒子间距的变化而重新计算合适的光滑长 度, 光滑长度与粒子的分布有密切的关系, 因此光滑 长度对时间的导数应遵循连续性方程变化规律:

$$
\frac{\mathrm{d}(h(t))}{\mathrm{d} t}=h(t) \operatorname{div}(v) .
$$

随着时间的推移, 每个粒子的光滑长度开始变得不 同, 当粒子相互远离时, 其光滑长度增加, 当粒子相 互靠近时, 其光滑长度也相应减少, 一般保持每个粒 子的邻域内所占有的邻粒子个数大致相当.

\section{3 初始质量分配}

在无网格计算中, Organ等人 ${ }^{[7]}$ 和Zhou等人 ${ }^{[8]}$ 利 用计算几何中的Voronoi多边形作为积分网格, 对于 SPH方法较难确定的每个粒子所占的空间体积, 此处 借用Voronoi图定义初始时刻每个粒子的质量. 当然 也可以采用规则排列的粒子, 特别对于立方体型的 模型, 但是对于较为复杂的区域或形状边界, Voronoi 图具有更好的适应性.

所谓 Voronoi 图, 即过相邻粒子的连线作垂直平 分线, 这些垂直平分线互相相交而构成的围绕粒子 的多边形, 如图 1. 根据 Voronoi 多边形的面积, 确定 每个粒子所负载的质量. 计算中, 每个粒子承载的质 量为 $m_{I}=\Delta S_{I} \times \Delta H \times \rho$, 其中 $\Delta S_{I}$ 是 Voronoi多边形面 积, $\Delta H$ 是每层粒子沿轴向所占的高度.

但是, 对于最外层的粒子, 无法按以上规则构筑 


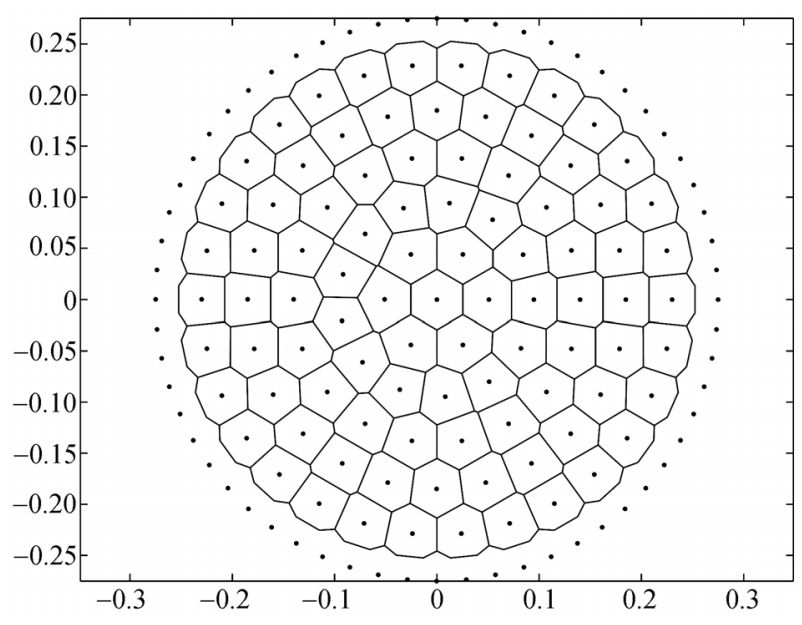

图 1 Voronoi 图的示意图

其 Voronoi 多边形, 因此, 实际建模时可在外围多布 置一层粒子, 然后截取所需要的粒子及体元结构.

在初始建模时, 应事先估算粒子的个数与分布, 尽量使每个粒子的排列间距均匀, 初始状况下粒子 排列的严重不均匀会对计算的稳定性和可靠性产生 不利影响.

\section{4 圆柱形铝液滴射流变形的数值模拟结果}

\section{1 模型和材料参数}

本文以铝液滴冲击刚性平面建立了 $\mathrm{SPH}$ 数值模 拟的模型: 假设铝液滴的形状是圆柱形, 半径为 0.24 $\mathrm{cm}$, 高为 $0.75 \mathrm{~cm}$, 以初始速度 $100 \mathrm{~m} / \mathrm{s}$ 的速度垂直撞 击刚性平面. 粒子模型如图 6(a), 沿长度方向分为 16 层, 每层圆截面上布置 92 个粒子, 共计 1472 个粒子. 液态铝在熔点温度附近黏度为 $1.18 \mathrm{mPa} \cdot \mathrm{s}$, 液态铝的 密度与固体时相差不大, 计算时约取 $2.5 \mathrm{~g} / \mathrm{cm}^{3}$, 计算 中不考虑铝液体与外界的热交换.

关于铝液的本构关系, 采用了 Gruneisen 状态方 程 ${ }^{[9]}$. 当材料压缩时:

$$
p=\frac{\rho_{0} C^{2} \mu\left[1+\left(1-\frac{\gamma_{0}}{2}\right) \mu-\frac{a}{2} \mu^{2}\right]}{\left[1-\left(S_{1}-1\right) \mu-S_{2} \frac{\mu^{2}}{\mu+1}-S_{3} \frac{\mu^{3}}{(\mu+1)^{2}}\right]}+\left(\gamma_{0}+a \mu\right) E,
$$

其中 $E$ 是单位体积内能; $C$ 是冲击波速度-粒子速度关 系式 $u_{\mathrm{s}}-u_{\mathrm{p}}$ 的曲线截距; $S_{1}, S_{2}$ 和 $S_{3}$ 是 $u_{\mathrm{s}}-u_{\mathrm{p}}$ 曲线斜率; $\gamma_{0}$ 是 Gruneisen 系数; $a$ 是关于 Gruneisen 系数与体
积关系的修正系数, 是一个材料常数; 压缩系数 $\mu=\frac{\rho}{\rho_{0}}-1$. 当材料膨胀时:

$$
p=\rho_{0} C^{2} \mu+\left(\gamma_{0}+a \mu\right) E .
$$

其各项参数取值如表 1 .

表 1 铝液的状态方程参数

\begin{tabular}{ccc}
\hline$S_{1}$ & $S_{2}$ & $S_{3}$ \\
\hline 1.339 & 0 & 0 \\
\hline \hline$C$ & $\gamma_{0}$ & $a$ \\
\hline 0.5386 & 1.97 & 0.48 \\
\hline
\end{tabular}

\section{2 计算结果图解和讨论}

图 2 给出了压力沿轴向传播过程, 图中红色一组 曲线反映了位于底面的编号为 2,21 和 63 粒子的压力 变化, 其对应的半径分别为 $0.05,0.14$ 和 $0.23 \mathrm{~cm}$. 绿 色和蓝色曲线分别是位于第 8 层和第 12 层相应半径 处的粒子压力变化过程, 可以看到在碰撞开始时, 底 面压力最先增加, 在随后的约 $1.5 \mu \mathrm{s}$ 内, 压力逐渐向 圆柱顶部传播, 并在传播过程中波形逐渐弥散, 压力 降低.

图 3 给出了底面上沿径向分布的粒子的速度变 化, 由内到外其编号分别为 $1,2,8,21,39$ 和 63 . 粒子 1 是位于底面轴线上的节点, 粒子 63 是位于底面周线 上的节点, 可以看到边缘上的液体最先开始向外流 动, 而靠近轴线部分仍保持压缩状态, 因此越靠近轴 线上的液体获得流动所需的时间越滞后于周线, 其 获得的流动速度也逐渐降低. 整个底面从碰撞开始 到进入稳定流动状态历时很短, 大约在 $0.7 \mu \mathrm{s}$ 以内.

此外, 采用有限元软件 LS-Dyna 计算了碰撞开 始后 $10.95 \mu \mathrm{s}$ 内的变形过程, 同样也反映了液体碰撞 后进入稳定流动的状态. 以粒子 8 为例, 分别比较了 有限元计算中相应节点处的径向位移和速度.

由图 4 和 5 可见, SPH 计算结果与 FEA 计算结 果符合得较好. 一般说来, 在网格划分和时间步长满 足要求的情况下, 有限元计算的结果应该是较为准 确的, 但对于本文问题, 在第 $30 \mu \mathrm{s}$ 以后, 由于单元 变形过大而导致精度降低甚至出现负体积的情况, 致使计算无法继续. 因此从这方面来说, SPH 方法具 有其特有的优势.

文中共计算了液柱与平面接触开始后 $90 \mu \mathrm{s}$ 内的 变形过程, 从不同时刻的变形图像中可以看到整个液 滴撞击刚性平面, 并在平面上发生流动的过程. 图 6 


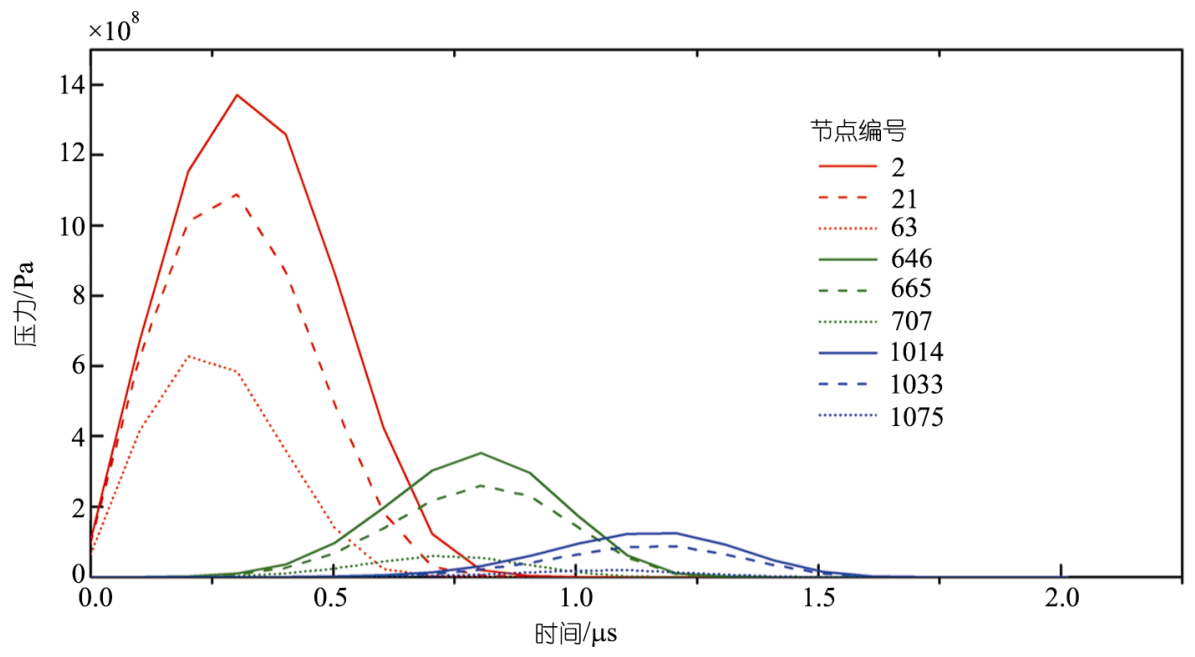

图 2 压力的传播过程

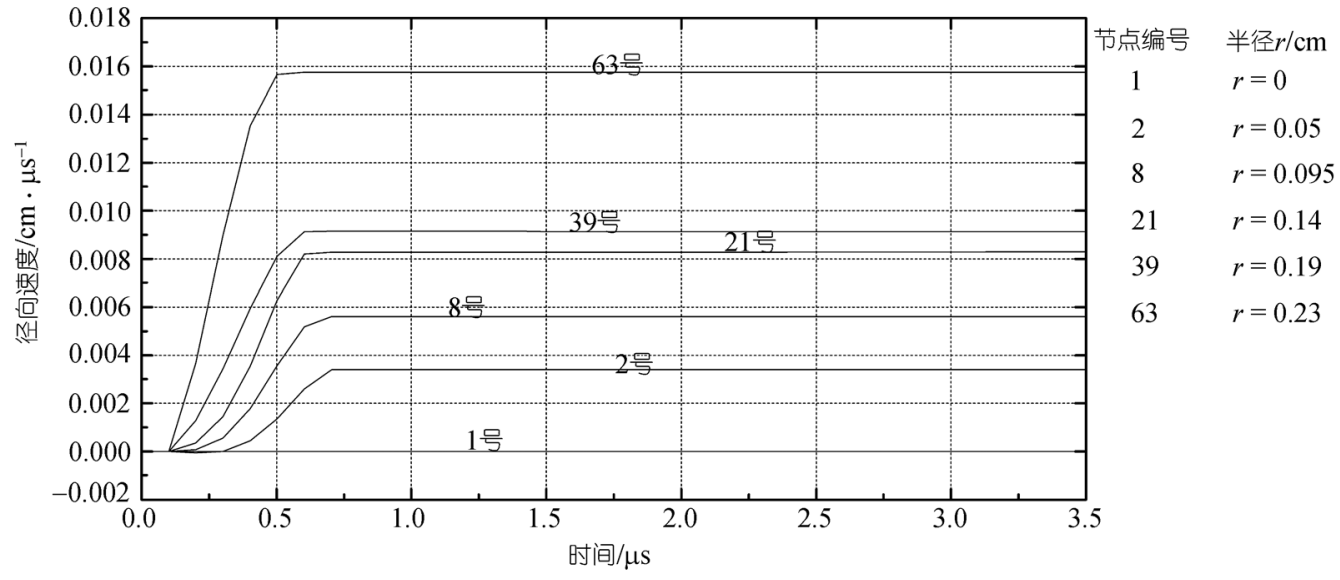

图 3 粒子短时程范围的径向速度变化

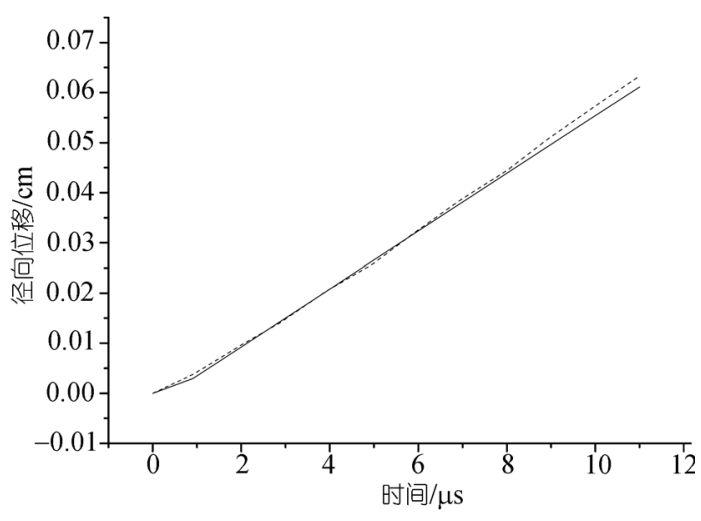

图 4 节点径向位移比较 实线示 SPH 计算结果; 虚线示 FEA 计算结果

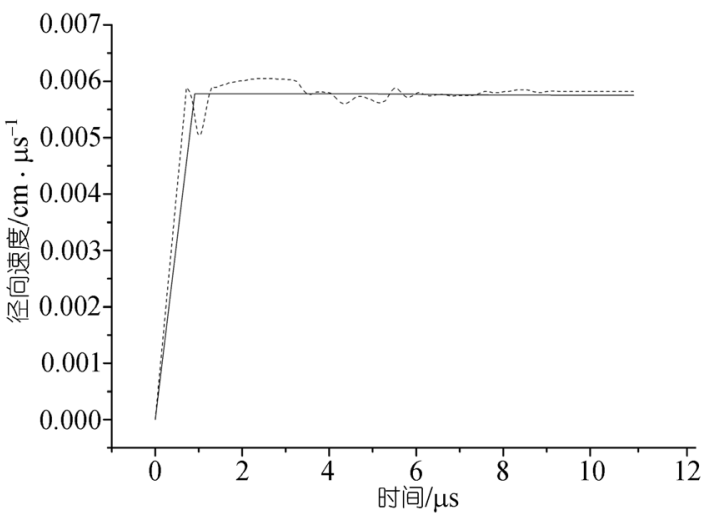

图 5 节点径向速度比较 实线示 SPH 计算结果; 虚线示 FEA 计算结果 
(a)

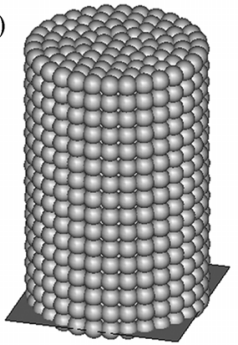

(b)

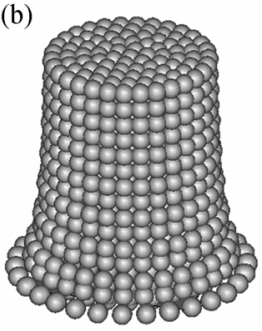

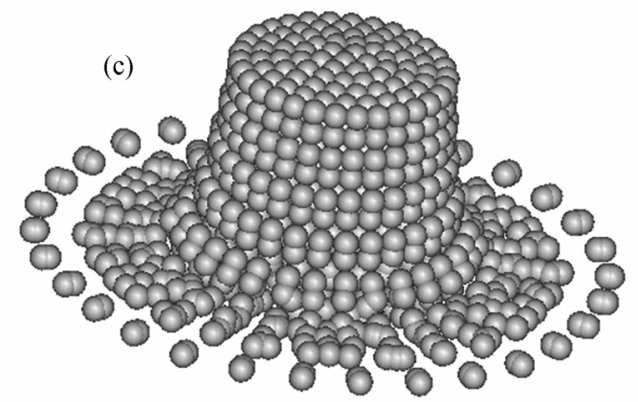
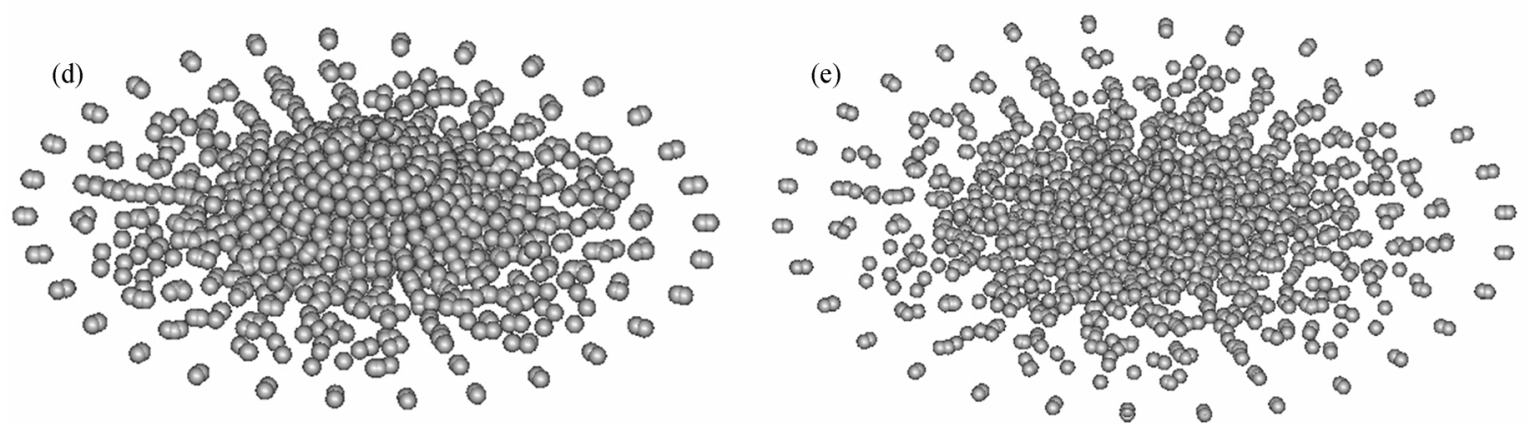

图 6 变形过程的图解说明

(a) $t=0 \mu \mathrm{s}$; (b) $t=9 \mu \mathrm{s}$; (c) $t=30 \mu \mathrm{s}$; (d) $t=50 \mu \mathrm{s}$; (e) $t=85 \mu \mathrm{s}$

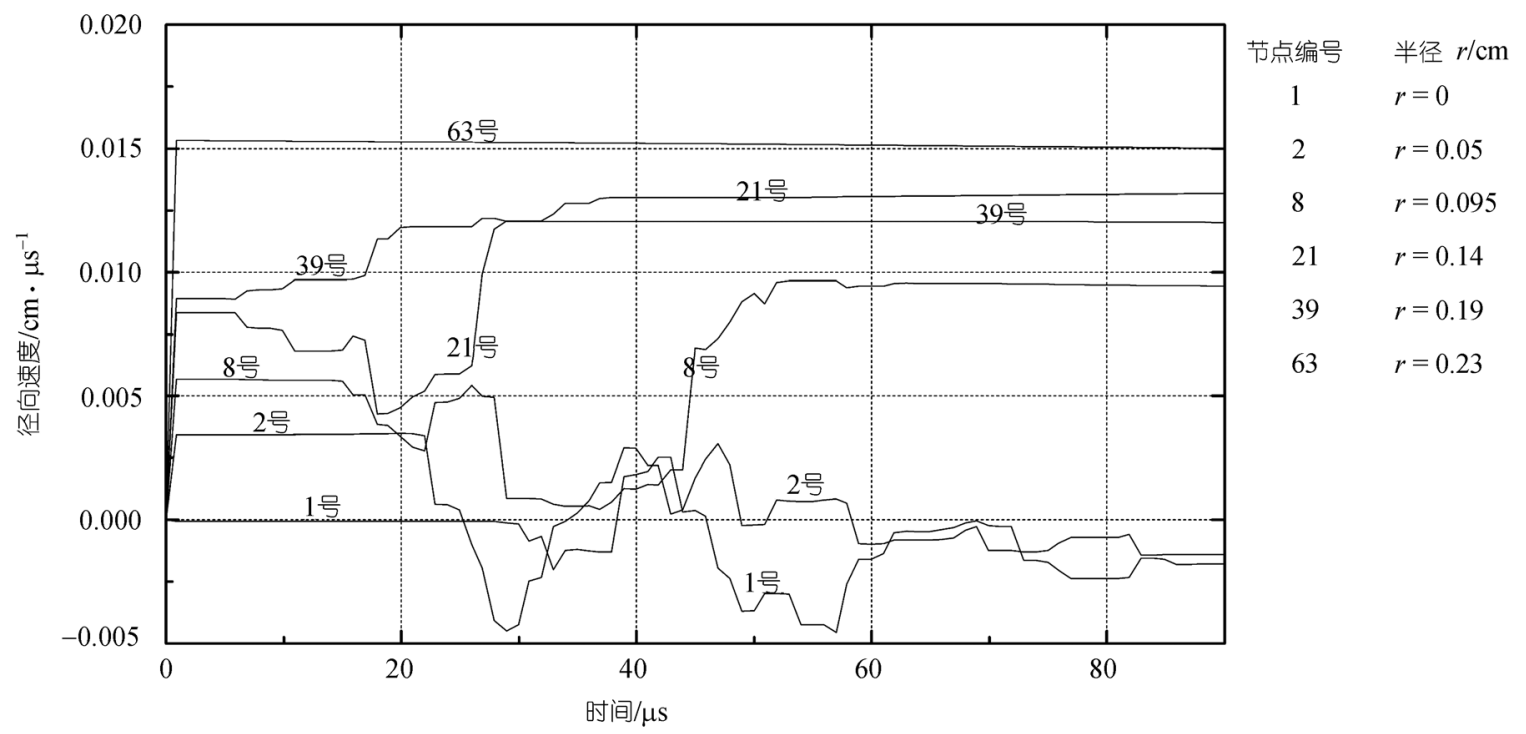

图 7 粒子长时程范围内径向速度变化

给出了不同时刻铝液柱的射流变形状况.

图 7 是整个计算时间范围内粒子的速度随时间 变化的曲线，从第 10 20 $\mu \mathrm{s}$ 开始，可以看到相邻粒子 的速度线由互相平行到互相纽结, 说明低速流体被 相邻的高速流体加速，而高速流体反之被减速，相互 之间存在动量的传递.
综上所述，液柱射流与刚性表面的碰撞经历了 3 个主要阶段：首先在 $0 \sim 0.7 \mu \mathrm{s}$ 时间内，底面的液体首 先完成了碰撞过程进入流动状态，在随后的 $0.7 \sim 10$ $\mu \mathrm{s}$ 时间内, 液体处于稳定流动状态; 其次约在第 10 60 $\mu \mathrm{s}$ 时间内, 流动逐渐开始变得混乱，相邻粒子 的速度线出现纠缠; 最后在 $60 \mu \mathrm{s}$ 以后, 速度线又发 
生分离，相邻粒子的速度也产生不同程度的增加或 降低，又进入了新的较为稳定的流动; 其中对于流动 过程中粒子速度线发生纠缠的现象究竟反映了流动 的本质还是由于 SPH 方法数值计算存在的误差造成 的, 尚需进一步研究.

\section{5 结论}

本文用 SPH方法研究了金属射流与刚性平面碰 撞过程，结果表明该方法可以有效地模拟液体(或固 体)的大变形, 且计算步骤简单明了, 易于编制程序. SPH方法中用一个作用于近程范围内的核函数来描 述粒子间相互作用的思想与分子动力学方法中镶嵌 电子云模型具有相似性 ${ }^{[10]}$. 这种方法除了在力学上 的应用外, 还可以应用到物理和化学等领域, 例如与 分子动力学模拟结合, 可以模拟带电粒子间的碰撞 以及化学反应动力学过程, 有可能开辟多尺度计算 的途径 ${ }^{[11]}$.

本文只考虑射流与刚性表面的碰撞，进一步的 工作还将包括与塑性材料的碰撞, 能够模拟射流对 材料的冲击效应和材料的形变过程, 射流冲击表面 时将激发冲击波，激波在物质中的传播将造成材料 内部的损伤, 这些都是有待模拟的问题. 模拟同样能 够在物理学中得到应用, 例如一粒子束打在靶物质 上, 粒子束被靶散射而飞溅，同时部分粒子穿透到靶 内部继续与内部物质碰撞, 在粒子能量较高时容易 激发激波, 会对靶产生压缩; 又如带电粒子之间的集 体碰撞过程也类似于本文所描述的问题，如果两类 粒子的质量相差很大, 则类似射流与表面的弹性碰
撞; 如果两类粒子质量相当, 则类似于射流与表面的 塑性碰撞. 因此 SPH 方法有着比较广阔的应用前景.

\section{参考文献}

1 Lucy L B. A numerical approach to the testing of fusion process. Astron J, 1977, 88: 1013-1024

2 Gingold R A, Monaghan J J. Smoothed particle hydrodynamics: Theory and application to non-spherical stars. Mon Not R Astron Soc, 1977, 181: 375-389

3 Johnson G R, Bsissel S R. Normalized smoothing functions for SPH impact computations. Int J Numer Math Eng, 1996, 39: 27252741[DOI]

4 Johnson G R, Stryk R A, Bsisse S R 1. SPH for high velocity impact computations. Comput Methods Appl Mech Eng, 1996, 139: 347-373[DOI]

5 Johnson G R, Bsissel S R, Stryk R A. A generalized particle algorithm for high velocity impact computations. Comput Mech, 2000, 25: 245-256[DOI]

6 Chen J K, Beraun J E, Jih C J. An improvement for tensile instability in smoothed particle hydrodynamics. Comput Mech, 1999, 23: 279-287[DOI]

7 Organ D, Fleming M, Terry T. Continous meshless approximations for nonconvex bodies by diffraction and transparency. Comput Mech, 1996, 18: 225-235

8 Zhou J X, Wen J B, Zhang H Y, et al. A nodal integration and post-processing technique based on Voronoi diagram for Galerkin meshless methods. Comput Methods Appl Mech Eng, 2003, 192(3536): $3831-3843$ [DOI]

9 ANSYS release 9.0 documentation: Material model examples. Canonsburg (PA): ANSYS Inc. 2004

10 Hoover W G. Isomorphism linking smoothed particles and embedded atoms. Physica A, 1998, 260: 244-254[DOI]

11 Hoover W G, Hoover C G. Links between microscopic and macroscopic fluid mechanics. Mol Phys, 2003, 101(11): 1559-1573[DOI] 